\title{
Expression signature of ten genes predicts the survival of patients with estrogen receptor positive-breast cancer that were treated with tamoxifen
}

\author{
HE HUANG, QIYU CHEN, WEIJIAN SUN, MINGDONG LU, YAOJUN YU, \\ ZHIQIANG ZHENG and PIHONG LI \\ Department of General Surgery, The Second Affiliated Hospital and \\ Yuying Children's Hospital of Wenzhou Medical University, Wenzhou, Zhejiang 325027, P.R. China
}

Received August 13, 2017; Accepted February 9, 2018

DOI: $10.3892 / \mathrm{ol} .2018 .8663$

\begin{abstract}
Although tamoxifen is the most frequently used drug for the treatment of estrogen receptor positive $\left(\mathrm{ER}^{+}\right)$-breast cancer (BRCA), its efficacy varies between patients. In the present study, Cox multivariate regression of the relative mRNA expression levels in two microarray-based datasets (GSE17005 and GSE26971) was employed to develop a risk score model to evaluate the outcome of patients with BRCA in the GSE17005 dataset. A total of ten genes were used to develop the prediction model for the survival of tamoxifen-treated patients with breast cancer. The survival time of patients in the low risk score group was significantly longer compared with patients in the high risk score group. This observation was validated in three other datasets (GSE26971, GSE22219 and GSE56884). The prognostic effect of the clinicopathological indicators and the risk score were tested with the 5-year event receiving operating characteristic curve, and the risk score had an improved prognostic value in patients with $\mathrm{ER}^{+}$-BRCA with an area under the curve value of 0.733 compared with the factors of age, tumor stage, tumor grade, chemotherapy, lymph invasion and tumor size. The risk score was significantly associated with the tumor-node-metastasis stage and grade, but was independent of age, sex, lymph invasion and tumor size. In summary, the risk model for breast cancer using the expression signature of ten genes may be an important indicator for predicting the survival of patients with $\mathrm{ER}^{+}$-breast cancer and treated with tamoxifen.
\end{abstract}

Correspondence to: Dr Pihong Li, Department of General Surgery, The Second Affiliated Hospital and Yuying Children's Hospital of Wenzhou Medical University, 109 West Xueyuan Road, Wenzhou, Zhejiang 325027, P.R. China

E-mail: lipihongwz@163.com

Key words: prognosis, breast cancer, tamoxifen

\section{Introduction}

Breast cancer (BRCA) is the most prevalent cancer type in women, with 74.1 new cases and 8.0 mortalities per 100,000 reported cases in developing countries in 2012 (1-3). Based on statistical estimation in China, 268,600 new breast cancer cases and 69,500 mortalities occurred due to BRCA in 2015 (4). Although the molecular subtyping of breast cancer is well developed and treatments are relatively abundant, many patients succumb to disease due to distant metastasis.

The estrogen receptor positive $\left(\mathrm{ER}^{+}\right)$subtype is currently the most curable breast cancer subtype. Tamoxifen, which binds to the ER and disrupts the ER signaling pathway, is the most popular treatment for the $\mathrm{ER}^{+}$-breast cancer. However, a large proportion of tamoxifen-treated $\mathrm{ER}^{+}$-patients still succumb to breast cancer (5). The current clinical staging system is insufficient for predicting the survival of the patients with $\mathrm{ER}^{+}$-breast cancer and treated with tamoxifen (6). Therefore, gene expression biomarkers of breast cancer are urgently needed for prognosis and treatment optimization.

According to previous studies, a single biomarker often fails to predict the outcome of the $\mathrm{ER}^{+}$-breast cancer across datasets, while models that are based on multiple genes significantly are more accurate (7). Therefore, in the present study, using Cox multivariate regression and data on gene expression levels, a risk score model for the prognosis of the $\mathrm{ER}^{+}$-breast cancer outcome in patients taking tamoxifen was developed using the GSE17005 dataset. Patients in the high-risk group exhibited a significantly higher 5-year survival rate compared with those in the low-risk group. Furthermore, this result was also replicated in four other independent cohorts (GSE26971, GSE22219,GSE42568 and GSE56884). According to the 5-year survival receiving operating characteristic (ROC), the area under the curve (AUC) of the risk score was higher compared with the other clinicopathological indicators in predicting the 5-year survival rate of the patients with $\mathrm{ER}^{+}$-breast cancer. The association between the clinicopathological indicators and the risk score was evaluated, and a nomogram describing the 5-year survival rate was also plotted. In summary, the risk score is a robust prognostic indicator for the survival of the patients with $\mathrm{ER}^{+}$-breast cancer and treated with tamoxifen. 


\section{Materials and methods}

Data pre-processing and sample selection. Samples in all datasets were filtered, and those without clear records of the $\mathrm{ER}^{+}$diagnosis or tamoxifen treatment were excluded from the dataset. The raw data containing the GSE17005, GSE26971, GSE22219, GSE42568 and GSE56884 datasets were downloaded using the raw data format from Gene Expression Omnibus (GEO, https://www.ncbi.nlm.nih.gov/geo/) according to the corresponding accession number. The samples that were either not from patients with $\mathrm{ER}^{+}$-breast cancer or treated with tamoxifen for five years were excluded from the present study. Subsequent to pre-processing, including normalization using Robust Multi-array Average (RMA), the data were used for further analysis. The probes and the gene names were matched, and the average values were calculated for genes that match the multiple probes. The Cancer Genome Atlas (TCGA) datasets were not used because the TCGA datasets were generated using RNA-seq platform while the other datasets were from microarray. The formula was dependent on the expression values (fragments per kb of transcript per million for RNA-seq and intensity for microarray), therefore the TCGA dataset was not used as training or test dataset in the present study.

Feature selection and model development. Cox univariate regression was implemented on GSE17005 and GSE26971 datasets. Genes that significantly associated with the survival rates in both datasets were selected as candidates. After 100 repeats and 100 iterations, the frequencies of genes were counted. Genes with the highest frequency were used for model development, and these 10 genes were identified. Multivariate Cox regression was then used to construct the linear risk score model in GSE17005. During the risk score calculation in the validation datasets, the coefficient for each gene was set to a constant value.

Statistical analysis. Statistical analyses were implemented using the R software (https://www.r-project.org/; version 3.0.1) and its packages. RMA normalization of the raw datasets was performed using the $\mathrm{R}$ function ' $r m a$ ' in the $\mathrm{R}$ package 'affy'. Cox univariate regression and multivariate regression was implemented using the package 'survival', and the ROC curve was plotted using functions in package 'pROC' (8).

\section{Results}

Candidate gene selection and model development. The workflow of the present study is illustrated in the Fig. 1A. Using the univariate Cox regression analysis, the association between the gene expression and the overall survival was calculated in 2 independent datasets: GSE17005 and GSE26971. A total of 48 genes that significantly associated with the survival rates in both datasets were identified as the candidate genes. Random forest variable hunting was conducted to retrieve the best combination of biomarkers, and ten genes were selected (Fig. 1B). The multivariate Cox regression analysis was implemented in GSE17005 instead of GSE26971 as the sample size of GSE17005 is bigger. The risk score was calculated using the following formula: Risk score $=(-0.382802602) \times$ NEK $2+$
$1.057608407 \times$ STC $2+0.216378311 \times$ CCNA $2+0.196075897$ $\mathrm{x}$ AK5 + 0.553253489 x CTDSP1 + (-0.544558722) x FOXD1 $+(-0.606202483) \times \mathrm{KCNK} 1+(-0.245868702) \times \mathrm{TNNC} 2+$ $(-0.093556696) \times$ CENPE $+(-0.212704033) \times$ STAT6. The coefficients are shown in Fig. 1C. The negative coefficient values indicate the tumor suppressor genes, and the positive values indicate the oncogenes for cancer development.

Prognostic value of the risk score in GSE17005. To evaluate the prognostic significance of the risk score for patients with $\mathrm{ER}^{+}$-breast cancer and treated with tamoxifen, the survival difference in patients from the high-risk and low-risk groups was analyzed. The median value of the risk score was used as a cutoff. The overall survival (OS) of patients in the high-risk group was significantly lower compared with the low-risk group (Fig. 2A, $\mathrm{P}=0.012$ ). As shown in Fig. 2B, patients in the high-risk group were characterized with early mortality, low expression levels of NIMA related kinase 2, cyclin A2 (CCNA2), forkhead box D1 (FOXD1), potassium two pore domain channel subfamily $\mathrm{K}$ member 1 and troponin $\mathrm{C}$ type 1 (slow), and high expression levels of stanniocalcin 2 (STC2), adenylate kinase 5 (AK5), carboxy-terminal domain RNA polymerase II polypeptide A small phosphatase 1 (CTDSP1) and signal transducer and activator of transcription 6 (STAT6). The ROC curve of 5-year survival was also plotted according to age, stage, grade, chemotherapy, lymph invasion, tumor size and risk score (Fig. 2C), and the area under the receiving operating characteristic curve was $0.676,0.622,0.631,0.663$, $0.618,0.596$ and 0.733 , respectively. Collectively, these results indicate that the risk score is a clinically important predictor of the 5-year survival of patients with $\mathrm{ER}^{+}$-breast cancer and treated with tamoxifen.

Validation of risk score performance. The good performance of the model in the training dataset may be due to over-fitness of the model to this dataset, particularly in multivariate analysis (9). Therefore, in order to evaluate this possibility, the risk scores for samples in four other datasets (GSE26971, GSE22219, GSE42568 and GSE56884) were calculated after the coefficients were fixed (Fig. 3). Similar to the survival profile in the training dataset, the survival of patients in the high-risk group was significantly poorer compared with the patients in the low-risk group in three of the four datasets $\left(\mathrm{P}=0.012,7.2 \times 10^{-10}\right.$ and 0.0068 for GSE26971, GSE22219 and GSE56884, respectively; Fig. 3A, B and D, top panel). The survival profile of the patients in the GSE42568 dataset was not significantly different between the two groups, which may be due to a smaller sample size. Similar expression trends were also observed in all four datasets (Fig. 3A, middle and bottom panel).

Risk score and clinicopathological indicators. Next, the association between the clinical observations and the risk score was calculated. The risk score was significantly associated with the breast cancer grade and the TNM stage. The risk score was independent from other clinical parameters (Fig. 4A). In order to compare the clinical significance and survival prediction of clinicopathological observations and the risk score, a nomogram of the 5-year survival rate was plotted (Fig. 4B). According to the nomogram, the risk score exhibited the widest range, indicating that it is an important prognostic indicator. 
A

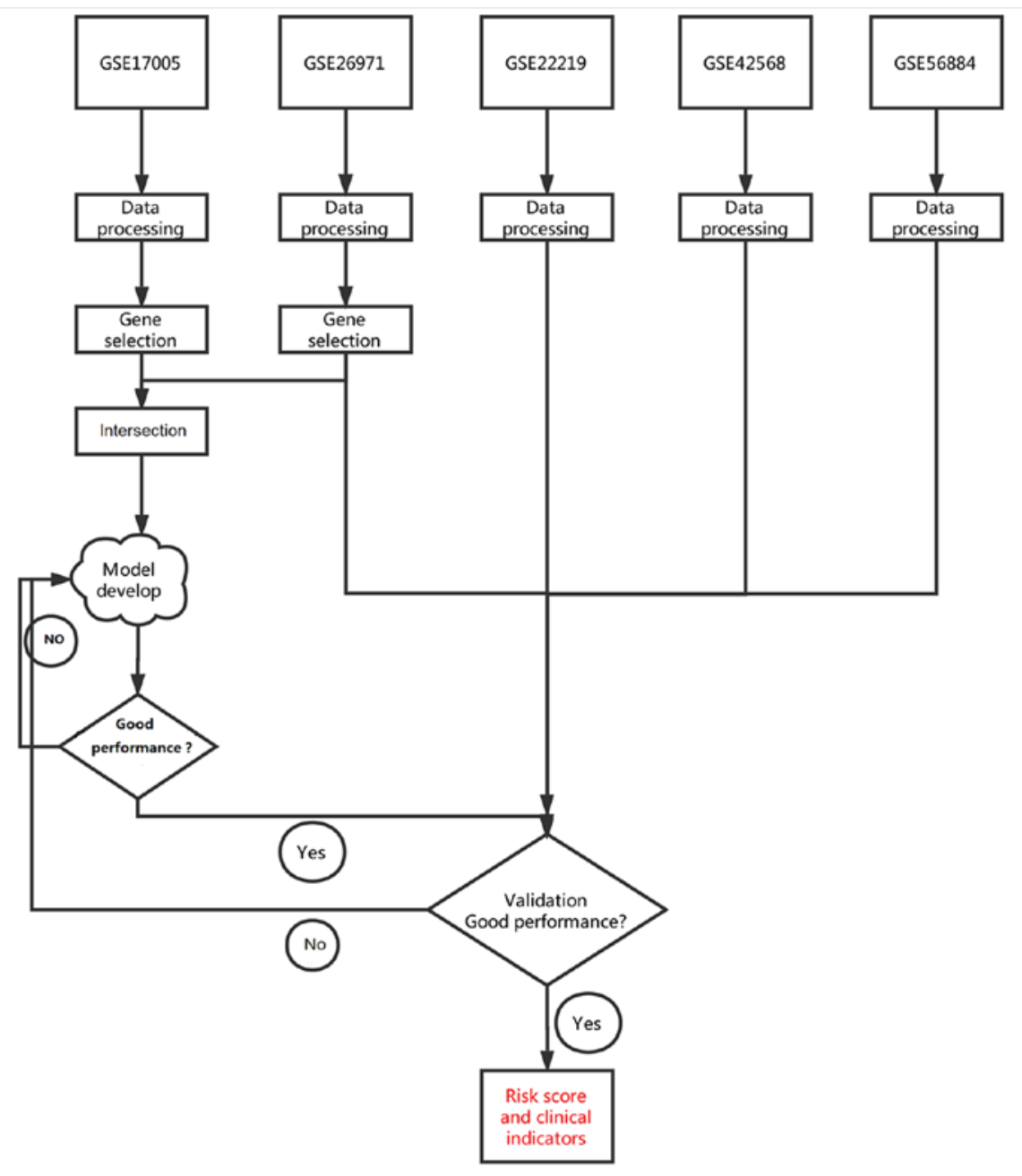

B

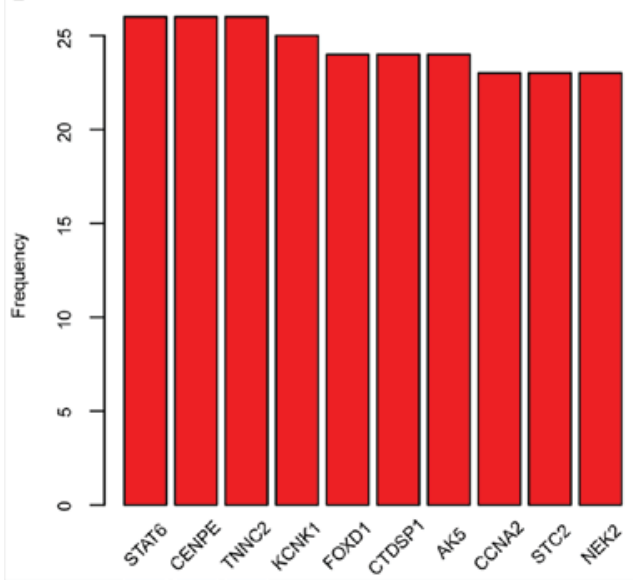

C

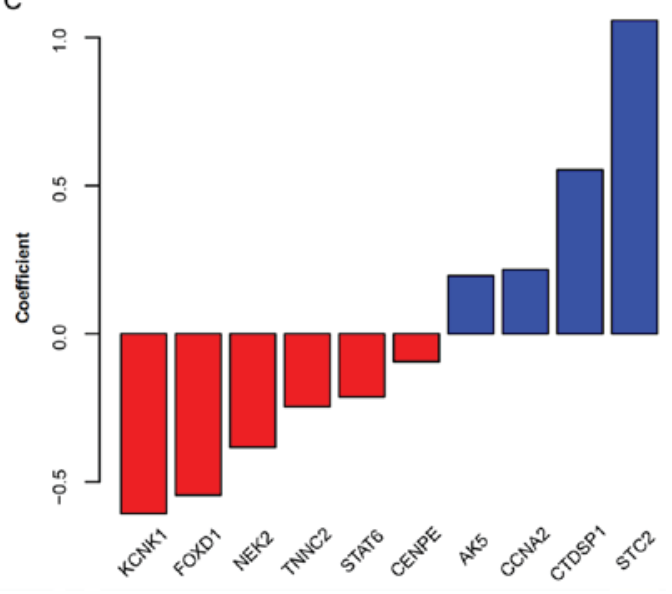

Figure 1. Workflow of the present study and candidate genes used for model development. (A) Workflow of the present study. (B) Frequency of genes in random forest variable hunting. (C) Coefficient for each gene in the risk score calculation formula. AK5, adenylate kinase 5; CCNA2, cyclin A2; CENPE, centromere protein E; CTDSP1, carboxy-terminal domain RNA polymerase II polypeptide A small phosphatase 1; FOXD1, forkhead box D1; KCNK1, potassium two pore domain channel subfamily K member 1 and troponin C type 1 (Slow); NEK2, NIMA related kinase 2; STAT6, signal transducer and activator of transcription 6; STC2, stanniocalcin 2; TNNC2, troponin C, skeletal muscle.

\section{Discussion}

Although the therapy of the $\mathrm{ER}^{+}$-breast cancer is relatively well-established to date and mostly consists of prescribing tamoxifen, the survival prognosis for patients with $\mathrm{ER}^{+}$-breast cancer and treated with tamoxifen varies, and clinicopathological observations remain insufficient $(10,11)$. Therefore, gene biomarkers for prognosis, drug selection and follow-up are urgently needed. Although many single molecular biomarkers for breast cancer prognosis have been studied in the past years, the clinical effect across datasets was observed to be limited, a multiple gene-based model is 


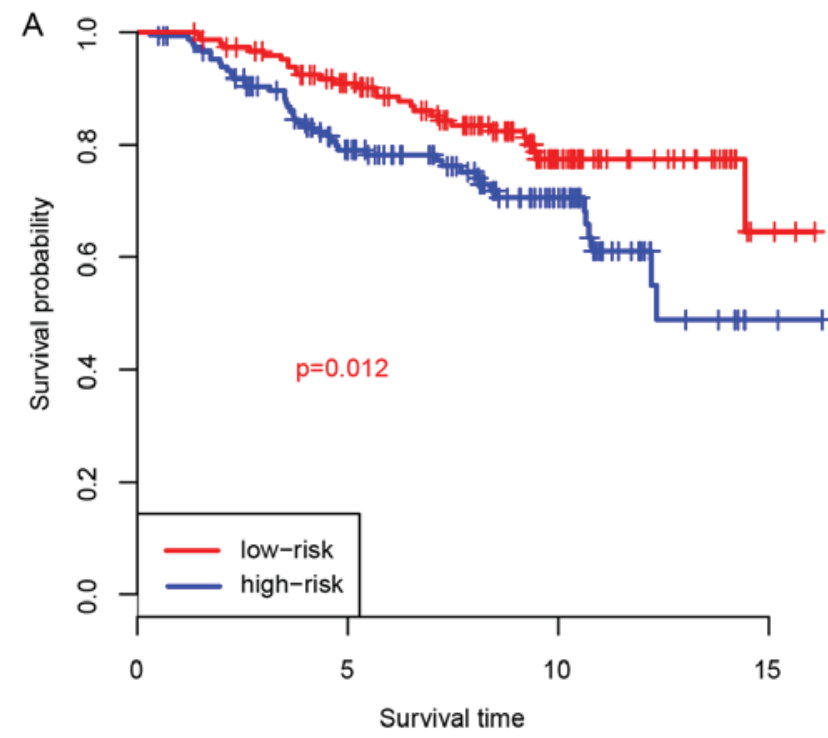

C

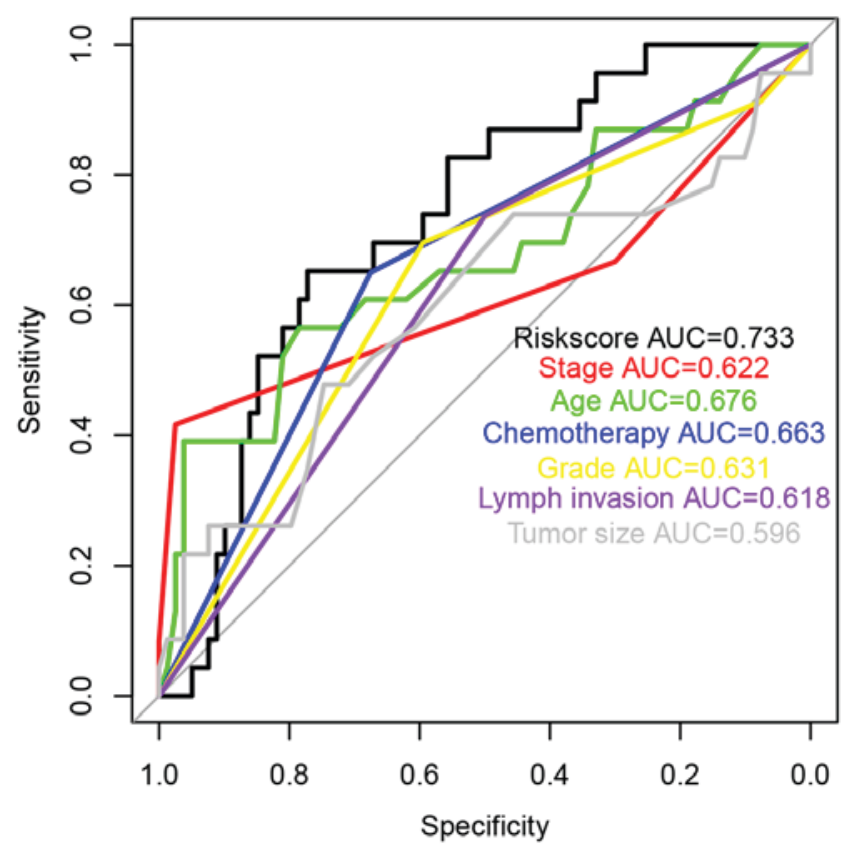

B
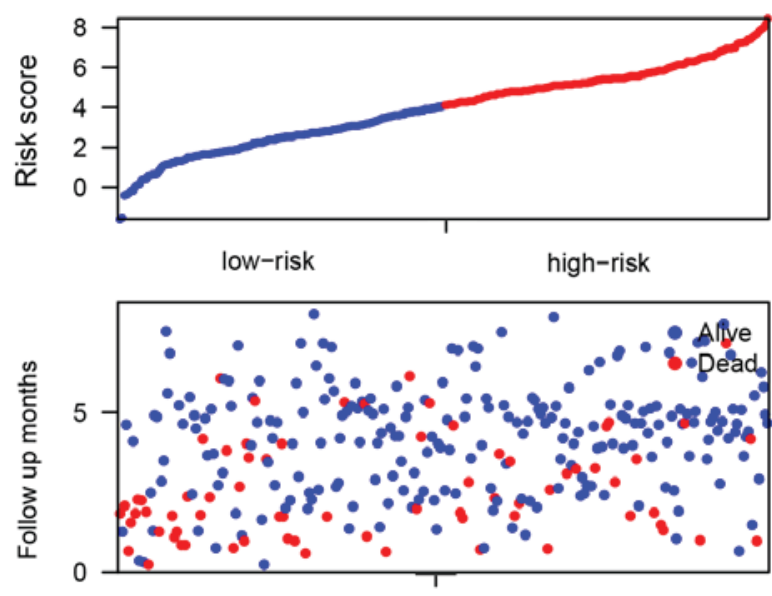

low-risk high-risk

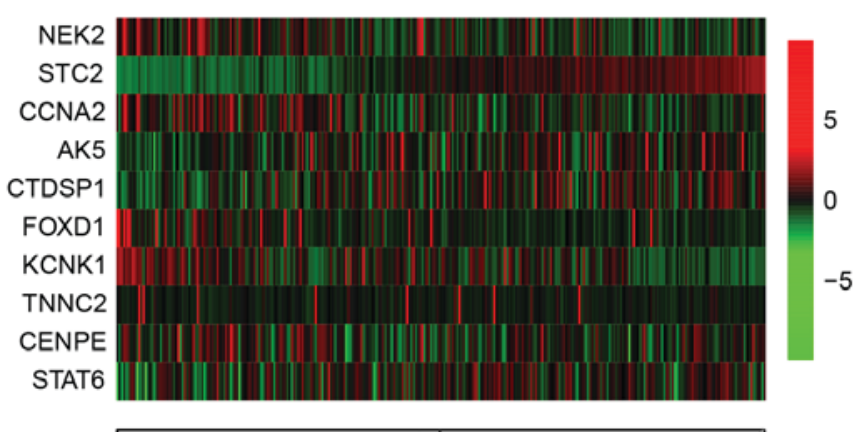

low-risk

high-risk

Figure 2. Risk score for prognosis in the training dataset. (A) Overall survival rate of the high- and low-risk groups. (B) The survival, mortality and the gene expression level in the high and low-risk groups. Red denotes upregulated gene expression levels. Green denotes downregulated gene expression levels. (C) 5-year survival receiving operating characteristic curves of the risk score and other clinical observations.

now preferred $(8,12-15)$. In the present study, Cox multivariate regression and random forest variable hunting were used on the GSE17005 dataset, and a risk score model for survival prediction was constructed. Patient groups with high and low risk scores were significantly different in terms of survival. Furthermore, this result was validated in three independent datasets. Compared with other clinicopathological indicators, the risk score is also an important prognostic indicator. Consistent with this, the value of 5-year survival ROC of the risk score is 0.733 , which is considerably higher compared with other clinical parameters (age, tumor stage, tumor grade, chemotherapy, lymph invasion and tumor size). In a previous study, tamoxifen-resistant biomarkers were identified using transcriptomic signatures. Among these genes, the highest 5-year relapse ROC was 0.64 for a single biomarker (16), while in the present study, it reached 0.733 , which indicates the high performance of the model.

Among these genes, STAT6 was previously shown to be associated with mortality and metastasis in breast cancer $(17,18)$. CENPE was reported to be associated with cell cycle (19), and FOXD1 was indicated to be associated with proliferation and drug resistance in breast cancer (20). CTDSP1 was reported to inhibit the migration and invasion of breast cancer cells (21). Aberrant methylation of AK5 was identified in breast cancer, although the mechanism is unclear (22). CCNA2 expression was associated with resistance to tamoxifen in $\mathrm{ER}^{+}$-breast cancer (23). STC2 

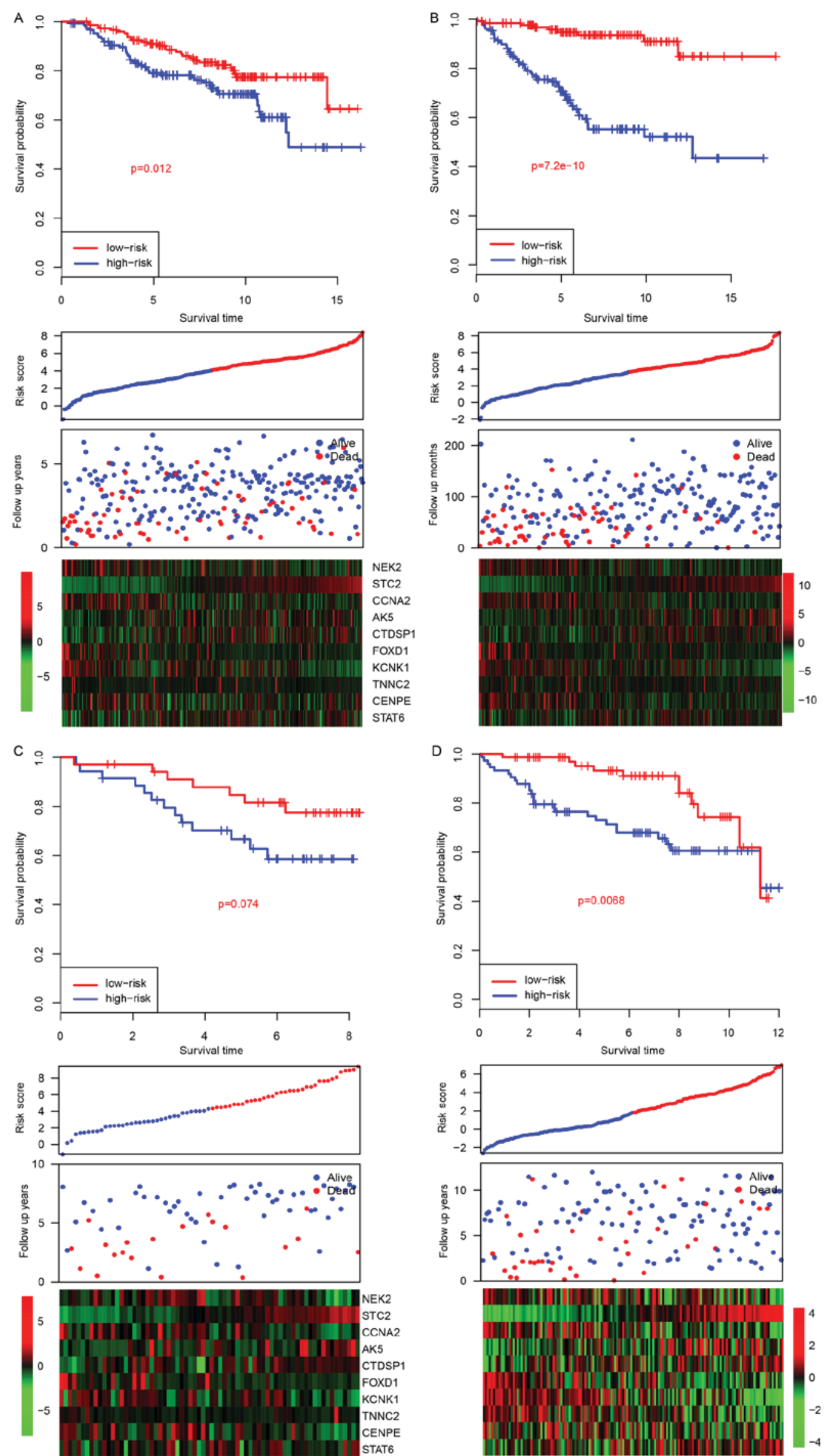

Figure 3. Impact of the risk score on survival in the validation datasets. Overall survival rate of the high-risk group and the low-risk group in four independent datasets: (A) GSE26971, (B) GSE22219, (C) GSE42568 and (D) GSE56884 (top panels). Detailed survival and expression information is provided in the middle and bottom panels. 
A

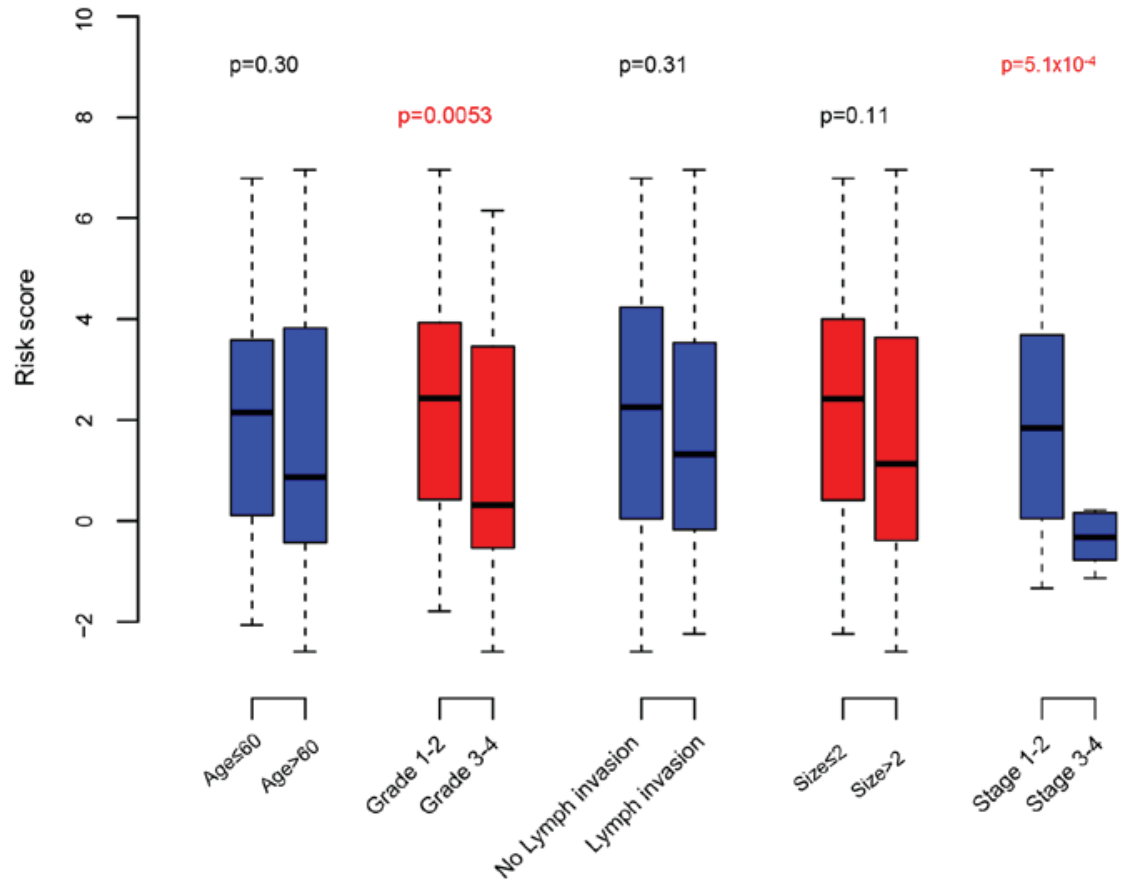

B

Points

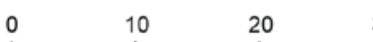

$30 \quad 40$

$50 \quad 60$

70

$80 \quad 90 \quad 100$

Risk score

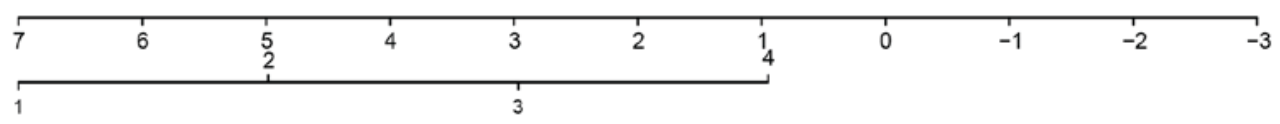

Stage

$\begin{array}{llllllllllllll}90,85 & 80 & 15 & 1 & 1 & 1 & 1 & 1 & 1 & 1 & 1 & 1 & 1 & 1\end{array}$

Chemo

0

Grade

Lymph

Size

Total Points

5 -year event rate

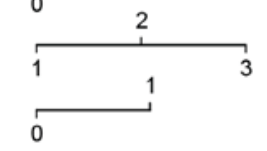

\begin{tabular}{lllllllll}
\hline 0 & 2 & 1 & 1 & 1 & 1 & 12 & 14 & 16
\end{tabular}

\begin{tabular}{|c|c|c|c|c|c|c|c|c|c|c|c|}
\hline 0 & 20 & 40 & 60 & 80 & 100 & 120 & 140 & 160 & 180 & 200 & 220 \\
\hline
\end{tabular}

Figure 4. Clinical information and risk score. (A) The association between the risk score and the clinicopathological information was evaluated. (B) A nomogram for 5-year survival. Lymph, lymph node metastasis; size, tumor size.

expression was associated with patient prognosis across different cancer types $(24,25)$. These reports indicate that the genes used for the development of this model are relatively reliable.

The present study has several limitations. The platform used in the four datasets is a microarray, which may limit the utilization of the risk score. Additionally, since the present study is a retrospective study, other epidemiological characteristics, clinical manifestations, pathological features and treatment methods of the samples were not assessed, and therefore a comprehensive analysis of the correlation scores between clinicopathological observations and risk score cannot be performed. Finally, the risk score formula was developed using the GSE17005 dataset, and a different formula was generated using the other datasets. It is difficult to justify which formula is better. In this article, the GSE17005 dataset was used for model development to minimize prediction error, and therefore bias may also exist.

\section{Acknowledgements}

Not applicable.

\section{Funding}

No funding was received.

\section{Availability of data and materials}

The data is available on Gene Expression Omnibus (https://www.ncbi.nlm.nih.gov/geo/).

\section{Author contributions}

HH, QC, WS and ML performed the experiments. YY, ZZ and $\mathrm{PL}$ analyzed the data. HH and PL were major contributors in 
the writing of the manuscript. All authors read and approved the final manuscript.

\section{Ethics approval and consent to participate}

All the experimental procedures were approved by the Ethics Committee of Wenzhou Medical University.

\section{Consent for publication}

Not applicable.

\section{Competing interests}

The authors declare that there are no competing interests.

\section{References}

1. Torre LA, Bray F, Siegel RL, Ferlay J, Lortet-Tieulent J and Jemal A: Global cancer statistics, 2012. CA Cancer J Clin 65: 87-108, 2015.

2. Huo Z, Gao Y, Yu Z, Zuo W and Zhang Y: Metastasis of breast cancer to renal cancer: Report of a rare case. Int J Clin Exp Pathol 8: 15417-15421, 2015.

3. Oztas E, Kara H, Kara ZP, Aydogan MU, Uras C and Ozhan G: Association between human telomerase reverse transcriptase gene variations and risk of developing breast cancer. Genet Test Mol Biomarkers 20: 459-464, 2016.

4. Siegel R, Miller K and Jemal A: Cancer statistics, 2015. CA Cancer J Clin 65: 5-29, 2015.

5. Takahashi M, Hayashida T, Okazaki H, Miyao K, Jinno H and Kitagawa Y: Loss of B-cell translocation gene 2 expression in estrogen receptor-positive breast cancer predicts tamoxifen resistance. Cancer Sci 105: 675-682, 2014.

6. Gonzalez-Angulo AM, Morales-Vasquez F and Hortobagyi GN: Overview of resistance to systemic therapy in patients with breast cancer. Adv Exp Med Biol 608: 1-22, 2007.

7. Salomaa V, Havulinna A, Saarela O, Zeller T, Jousilahti P, Jula A, Muenzel T, Aromaa A, Evans A, Kuulasmaa K and Blankenberg S: Thirty-one novel biomarkers as predictors for clinically incident diabetes. PLoS One 5: e10100, 2010.

8. Robin X, Turck N, Hainard A, Tiberti N, Lisacek F, Sanchez JC and Müller M: pROC: An open-source package for R and S+ to analyze and compare ROC curves. BMC Bioinformatics 12: 77, 2011.

9. Zhang Z: Too much covariates in a multivariable model may cause the problem of overfitting. J Thorac Dis 6: E196-E197, 2014.

10. Apuri S: Neoadjuvant and adjuvant therapies for breast cancer. South Med J 110: 638-642, 2017.

11. Glassman D, Hignett S, Rehman S, Linforth R and Salhab M: Adjuvant endocrine therapy for hormone-positive breast cancer, focusing on ovarian suppression and extended treatment: An update. Anticancer Res 37: 5329-5341, 2017.

12. Gogalic S, Sauer U, Doppler S, Heinzel A, Perco P, Lukas A, Simpson G, Pandha H, Horvath A and Preininger C: Validation of a protein panel for the non-invasive detection of recurrent non-muscle invasive bladder cancer. Biomarkers 22: 674-681, 2017.
13. Urquidi V, Netherton M, Gomes-Giacoia E, Serie DJ, Eckel-Passow J, Rosser CJ and Goodison S: A microRNA biomarker panel for the non-invasive detection of bladder cancer. Oncotarget 7: 86290-86299, 2016.

14. Li Y, Huang J, Sun J, Xiang S, Yang D, Ying X, Lu M, Li H and Ren G: The transcription levels and prognostic values of seven proteasome alpha subunits in human cancers. Oncotarget 8: 4501-4519, 2017

15. Kavalieris L, O'Sullivan P, Frampton C, Guilford P, Darling D, Jacobson E, Suttie J, Raman JD, Shariat SF and Lotan Y: Performance characteristics of a multigene urine biomarker test for monitoring for recurrent urothelial carcinoma in a multicenter study. J Urol 197: 1419-1426, 2017.

16. Mihaly Z, Kormos M, Lanczky A, Dank M, Budczies J, Szász MA and Győrffy B: A meta-analysis of gene expression-based biomarkers predicting outcome after tamoxifen treatment in breast cancer. Breast Cancer Res Treat 140: 219-232, 2013.

17. Slattery ML, Lundgreen A, Hines LM, Torres-Mejia G, Wolff RK, Stern MC and John EM: Genetic variation in the JAK/STAT/SOCS signaling pathway influences breast cancer-specific mortality through interaction with cigarette smoking and use of aspirin/NSAIDs: The breast cancer health disparities study. Breast Cancer Res Treat 147: 145-158, 2014.

18. Papageorgis P, Ozturk S, Lambert AW, Neophytou CM, Tzatsos A, Wong CK, Thiagalingam S and Constantinou AI: Targeting IL13Ralpha2 activates STAT6-TP63 pathway to suppress breast cancer lung metastasis. Breast Cancer Res 17: 98 , 2015.

19. Hou S, Li N, Zhang Q, Li H, Wei X, Hao T, Li Y, Azam S, Liu C, Cheng $\mathrm{W}$, et al: $\mathrm{XAB} 2$ functions in mitotic cell cycle progression via transcriptional regulation of CENPE. Cell Death Dis 7: e2409, 2016.

20. Zhao YF, Zhao JY, Yue H, Hu KS, Shen H, Guo ZG and Su XJ: FOXD1 promotes breast cancer proliferation and chemotherapeutic drug resistance by targeting p27. Biochem Biophys Res Commun 456: 232-237, 2015

21. Sun T, Fu J, Shen T, Lin X, Liao L, Feng XH and Xu J: The small c-terminal domain phosphatase 1 inhibits cancer cell migration and invasion by dephosphorylating ser(p)68-twist1 to accelerate twist1 protein degradation. J Biol Chem 291: 11518-11528, 2016.

22. Miyamoto K, Fukutomi T, Akashi-Tanaka S, Hasegawa T, Asahara T, Sugimura T and Ushijima T: Identification of 20 genes aberrantly methylated in human breast cancers. Int J Cancer 116: 407-414, 2005.

23. Gao T, Han Y, Yu L, Ao S, Li Z and Ji J: CCNA2 is a prognostic biomarker for ER+ breast cancer and tamoxifen resistance. PLoS One 9: e91771, 2014.

24. Arigami T, Uenosono Y, Ishigami S, Yanagita S, Hagihara T, Haraguchi N, Matsushita D, Hirahara T, Okumura H, Uchikado $\mathrm{Y}$, et al: Clinical significance of stanniocalcin 2 expression as a predictor of tumor progression in gastric cancer. Oncol Rep 30: 2838-2844, 2013.

25. Jansen MP, Sas L, Sieuwerts AM, Van Cauwenberghe C, Ramirez-Ardila D, Look M, Ruigrok-Ritstier K, Finetti P, Bertucci F, Timmermans MM, et al: Decreased expression of ABAT and STC2 hallmarks ER-positive inflammatory breast cancer and endocrine therapy resistance in advanced disease. Mol Oncol 9: 1218-1233, 2015. 